Portland State University

PDXScholar

\title{
An experimental investigation of the finite time efficiency of a Peltier refrigeration device
}

Thomas Schneider

Portland State University

Follow this and additional works at: https://pdxscholar.library.pdx.edu/open_access_etds

Part of the Physics Commons, and the Thermodynamics Commons

Let us know how access to this document benefits you.

\section{Recommended Citation}

Schneider, Thomas, "An experimental investigation of the finite time efficiency of a Peltier refrigeration device" (1991). Dissertations and Theses. Paper 4261.

https://doi.org/10.15760/etd.6145

This Thesis is brought to you for free and open access. It has been accepted for inclusion in Dissertations and Theses by an authorized administrator of PDXScholar. Please contact us if we can make this document more accessible: pdxscholar@pdx.edu. 
AN ABSTRACT OF THE THESIS OF Thomas Schneider for the Master of Science in Physics presented August 14, 1991.

Title: An Experimental Investigation of the Finite Time Efficiency of a Peltier Refrigeration Device.

APPROVED BY THE MEMBERS OF THE THESIS COMMITTEE:

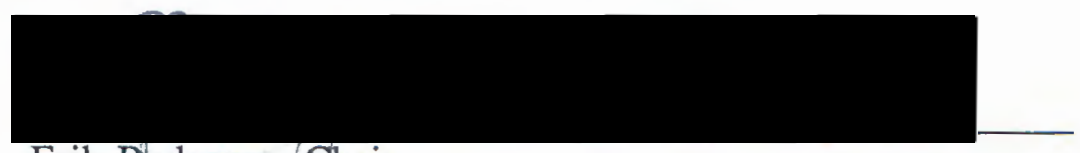

Erik Bpodegom, Ghair

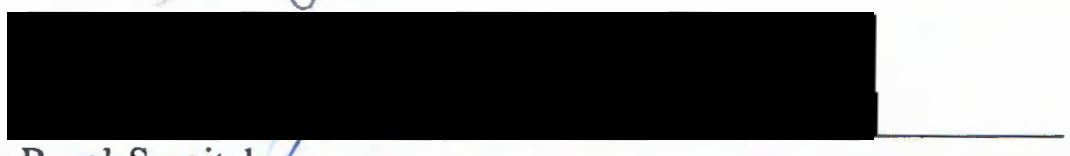

Pavel Smejtek

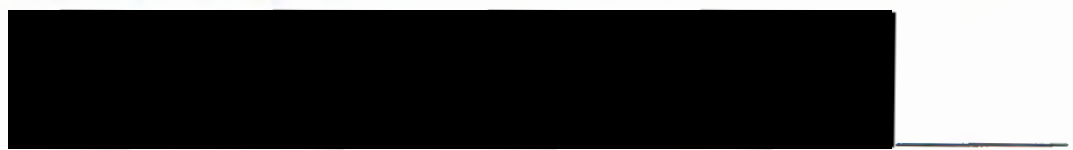

Laird Brodie

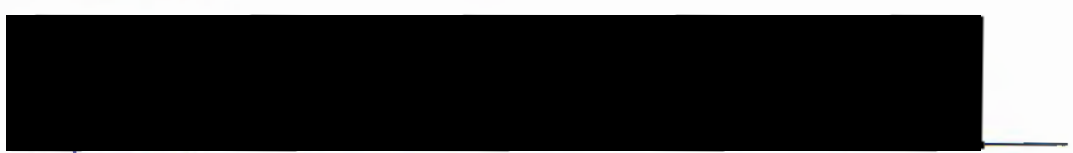

Neal DeChillo

Since the need of energy conservation has become more and more urgent in the past decades, there has been an increased interest in the study and development of more efficient energy conversion systems. One of the fields that have arisen from that endeavor is a branch of physics called Finite Time Thermodynamics (FTT). It may be said that FTT was initiated through the famous paper by Curzon and Ahlborn (1975) that established new bounds on the efficiency of a finite time Carnot heat engine. Before, the 
traditional treatments gave a fundamental upper limit on the efficiency of any heat engine. However, this figure, the well-known Carnot efficiency, is far too optimistic in comparison to real heat engines. The reason lies in the fact that the traditional Carnot engine is operating infinitely slowly, thus having zero power output. Curzon and Ahlborn were able to improve upon this treatment and to set an upper limit on engines producing finite power.

In 1990, Joseph D. Walters (PSU) presented a new bound on the efficiency of a finite time Carnot refrigeration cycle at maximum cooling power. In addition he gave the relation between efficiency and cycle frequency and between cooling power and cycle frequency.

The purpose of the present work was to develop an experimental set-up that allows for a check of these theoretical results. For convenience of measurement, a thermoelectric refrigeration or Peltier device seemed to be the most practical, since the input power is readily measurable.

The project encompassed the physical set-up of the experiment, the development of electronic units that allow the experiment to be interfaced with a microcomputer, and the writing of a computer program that organizes control and measurement procedures. 


\title{
AN EXPERIMENTAL INVESTIGATION OF THE FINITE TIME EFFICIENCY OF A PELTIER REFRIGERATION DEVICE
}

\author{
by
}

THOMAS SCHNEIDER

A thesis submitted in partial fulfillment of the requirements for the degree of

\author{
MASTER OF SCIENCE \\ in \\ PHYSICS
}

Portland State University

1991 
TO THE OFFICE OF GRADUATE STUDIES:

The members of the Committee approve the thesis of Thomas Schneider presented August 14, 1991.

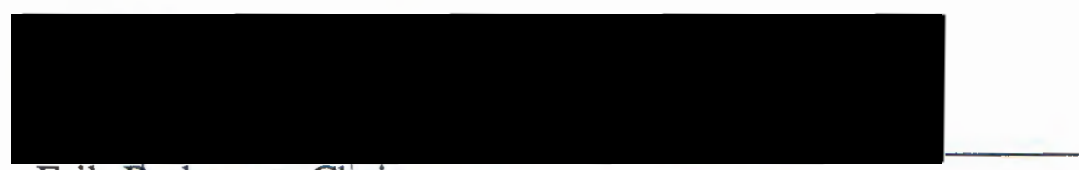

Erik Bodegom, Chair

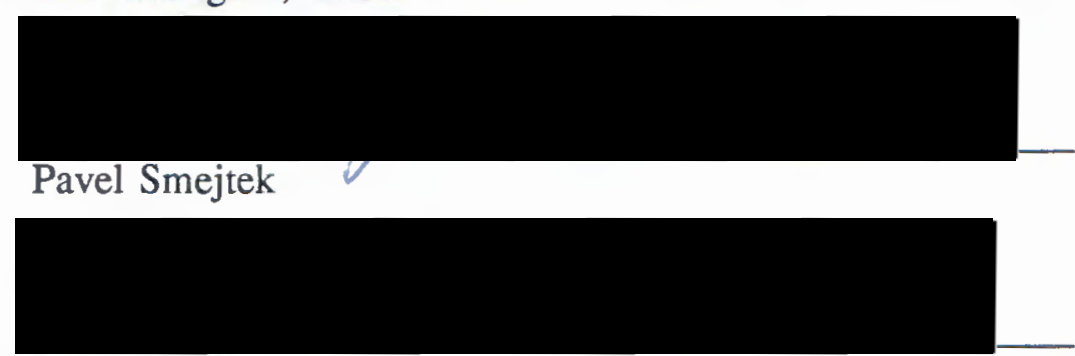

Laird Brodie

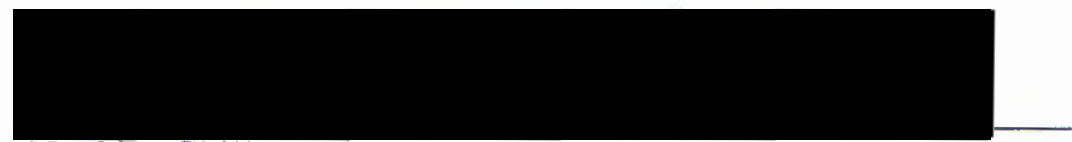

Neal DeChillo

\section{APPROVED:}

Mark Gurevitch, Chair, Department of Physics

C. William Savery, Interim Vice Provost tor Graduate Studies and Research 


\section{ACKNOWLEDGEMENTS}

For the ever patient, friendly, and helpful guidance through the various vicissitudes of this project I would like to thank my advisor, Dr. Erik Bodegom, who enabled me with his enormous experience in the fields of electronics and computer interfacing to design and build the present experiment. Furthermore I want to express my gratitude towards Rudolf Zupan of the machine shop who was always open for the many details the construction of the experiment required. Finally, my friend Dmitry Sharkov deserves mentioning, who gave me great support in all questions concerning the technical aspects of writing the thesis paper, including the use of word processors. 
TABLE OF CONTENTS

PAGE

ACKNOWLEDGEMENTS $\ldots \ldots \ldots \ldots \ldots \ldots \ldots \ldots$ iii

LIST OF FIGURES $\quad \ldots \ldots \ldots \ldots \ldots \ldots \ldots \ldots$ vi

\section{CHAPTER}

I INTRODUCTION $\ldots \ldots \ldots \ldots \ldots \ldots \ldots \ldots \ldots$

II THERMOELECTRICITY $\ldots \ldots \ldots \ldots \ldots \ldots \ldots$

Description of Thermoelectric Effects ......... 3

Theory of Thermoelectric Refrigeration ......... 5

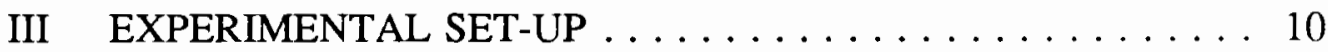

General Description of the Experiment $\ldots \ldots \ldots \ldots 10$

Principle of Heat Flow Measurement . . . . . . . 13

Thermocouples $\ldots \ldots \ldots \ldots \ldots \ldots \ldots \ldots \ldots \ldots$

Temperature Measurements with Thermistors . . . . . . 15

Electronic Circuitry . . . . . . . . . . . . 17

Purpose of the Control Unit

Description of the Components of the Control Unit

Computer Program $\ldots \ldots \ldots \ldots \ldots \ldots \ldots \ldots \ldots$

Comparison with the PASCO efficiency apparatus . . . . 22

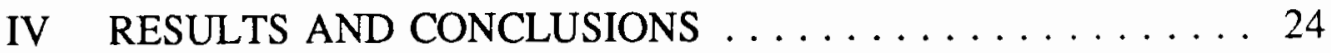




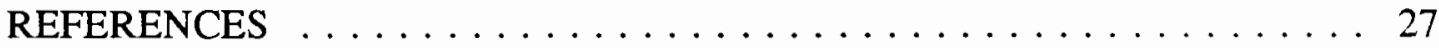

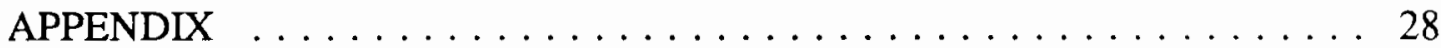




\section{LIST OF FIGURES}

FIGURE

PAGE

1. Circuit Used in Defining the Thermoelectric Coefficients .... 3

2. Simple Thermoelectric Refrigerator $\ldots \ldots \ldots \ldots \ldots$

3. Cooling Power of a Peltier Element

versus Dimensionless Current $\ldots \ldots \ldots \ldots \ldots$

4. Reduced Coefficient of Performance of a Peltier Element versus Dimensionless Current $\ldots \ldots \ldots \ldots \ldots \ldots$

5. Schematic Diagram of the Experimental Set-Up . . . . . . . 12

6. Basic Configuration of the Type-K Thermocouple ........ 16

7. Thermocouple Configuration to Measure Temperature Differences $\ldots \ldots \ldots \ldots \ldots \ldots \ldots$

8. Schematic Diagram of the Control Unit $\ldots \ldots \ldots \ldots \ldots$

9. Schematic Diagram of the Amplifier Circuit $\ldots \ldots \ldots \ldots$ 


\section{CHAPTER I}

\section{INTRODUCTION}

It is a well-known fact that the consumption of energy on our planet keeps increasing whereas it is not clear at all if and how this increasing demand of an exponentially growing world population can be satisfied in the future. While great efforts have been and still are being made in researching new of sources of energy, the search for methods of energy conservation and for more efficient means of energy conversion must be considered to be equally important. And indeed, there has been an increased interest in the study and development of more efficient energy conversion systems. One of the fields that emerged from these endeavors is a branch of physics named Finite Time Thermodynamics (FTT). It may be said that FTT was initiated in 1975 through the famous paper by Curzon and Ahlborn (1) of the University of British Columbia. Their work concerned itself with an improved model of a real Carnot heat engine. Before, the traditional treatments tried to apply equilibrium thermodynamics in order to obtain theoretical descriptions of the performance characteristics of real heat engines. One of the most famous results of the traditional treatments is the fundamental upper bound on the efficiency of any heat engine operating between two temperature reservoirs with temperatures $T_{H}$ and $T_{C}$, respectively, the Carnot efficiency $\eta_{\text {Carnot }}=1-T_{C} / T_{H}$. The disadvantage of this result lies in the fact that it is based on the assumption that the two adiabatic and two isothermal branches of the cycle are traversed by moving from one 
point of equilibrium to another. But this could only take place in infinite time spans. The consequence is that a Carnot cycle cannot yield any power output and it turns out that the Carnot efficiency is far too optimistic for real heat engines operating in finite time. Real engines are suffering losses through irreversible effects such as finite-rate heat transfer, friction, and heat leaks and hence their efficiency lies well below the Carnot limit.

The same considerations can also be applied to refrigeration cycles and in 1990, Joseph D. Walters (2) of Portland State University presented a new bound on the efficiency of a finite time Carnot refrigeration cycle running at a certain operation mode. In his dissertation he also gave the relation between efficiency and cycle frequency and between cooling power and cycle frequency.

The purpose of the present work was to develop an experiment that allows for a check of these theoretical results. Since thermoelectric devices are relatively well accessible to measurements and experimental control, a Peltier element was chosen to this end.

The project encompassed the physical set-up of the experiment, the development of electronic units that allow the experiment to be interfaced with a microcomputer, and the design of computer software that organizes various control and measurement procedures. 


\section{CHAPTER II}

\section{THERMOELECTRICITY}

\section{DESCRIPTION OF THERMOELECTRIC EFFECTS}

The first discovery of a thermoelectric effect took place in 1821 when Thomas Seebeck realized that an electromotive force could be produced by heating a junction between two dissimilar metals. Thirteen years later the French scientist Jean Peltier discovered that the passage of an electric current through the junction of two dissimilar metals caused cooling of one side and in 1838 , Heinrich Lenz was actually able to freeze a droplet of water using the Peltier effect. In 1855, William Thomson derived the relationship between these two effects from thermodynamic principles and he predicted another thermoelectric effect, that had then been unknown.

Referring to Figure 1, the three thermoelectric coefficients will now be defined.

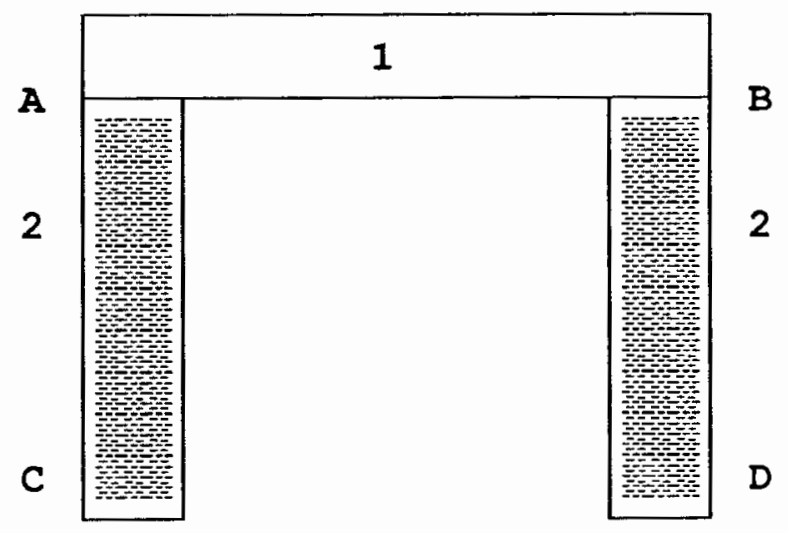

Figure 1. Circuit used in defining the thermoelectric coefficients. 
Figure 1 shows a circuit consisting of two conductors 1 and 2 with two junctions $\mathrm{A}$ and $\mathrm{B}$. If there is a temperature difference $\Delta \mathrm{T}$ between $\mathrm{A}$ and $\mathrm{B}$ then a potential difference V can be measured between $\mathrm{C}$ and D. The differential Seebeck coefficient is then defined as

$$
\alpha_{12}=\frac{V}{\Delta T}
$$

If in the same configuration a current $I$ is flowing through the circuit in the suitable direction, there will be heating at the junction $\mathrm{A}$ and cooling at junction $\mathrm{B}$, both at the same rate $\mathrm{q}$. The differential Peltier coefficient is then defined as

$$
\Pi_{12}=\frac{q-I^{2} R_{J}}{I}
$$

where the Joule heat at the junction with resistance $R_{J}$ has been subtracted.

A temperature gradient $\mathrm{dT} / \mathrm{dx}$ in addition to a current in either one of the conductors leads to a rate of heating or cooling $\mathrm{dq} / \mathrm{dx}$ per unit length. The coefficient of proportionality for this effect, the Thomson effect is given by

$$
\tau=\frac{\frac{d q}{d x}}{I \frac{d T}{d x}}
$$

The following relations, now known as Kelvin relations were deduced by Thomson through application of the first and second laws of thermodynamics to the simple circuit above under the assumption of reversibility. Although this assumption is questionable, as Thomson was well aware of, an application of the theory of irreversible thermodynamics leads to the same equations (1.4) and (1.5). 


$$
\Pi_{12}=\alpha_{12} T
$$

and

$$
\tau_{1}-\tau_{2}=T \frac{d \alpha_{12}}{d T}
$$

While the Thomson coefficient has been defined above for just one single material, the two other coefficients have been defined for junctions of two materials. It is convenient to introduce absolute Peltier and Seebeck coefficients for single materials.The differential coefficients can then be obtained as the difference between two absolute coefficients.

\section{THEORY OF THERMOELECTRIC REFRIGERATION}

Figure 2 shows a single thermoelectric element with branches possessing the material constants $\alpha_{1}, \sigma_{1}, \kappa_{1}$ and $\alpha_{2}, \sigma_{2}, \kappa_{2}$, respectively, where $\alpha$ is the absolute Seebeck coefficient, $\sigma$ is the electrical conductivity, and $\kappa$ is the thermal conductivity. The two branches are of constant cross-sectional areas $A_{1}$ and $A_{2}$ and have lengths $l_{1}$ and $l_{2}$. The branches are connected by a link of zero resistance at the heat source and by a source of electromotive force, which produces a current I, at the heat sink. The temperatures of source and sink are $T_{C}$ and $T_{H}$ respectively. The whole thermoelectric element is considered to be a thermally isolated system.

The coefficient of performance (COP) of a refrigeration device is defined as the quotient of the heat flow rate at the heat source $\mathrm{q}_{\mathrm{C}}$ and the power input into the system. 
For refrigerators with discrete cycles, an alternative definition would be the ratio of cooling work per cycle to the electrical work expended per cycle. In the following the COP of a Peltier refrigeration element like the one shown in Figure 2 will be derived.

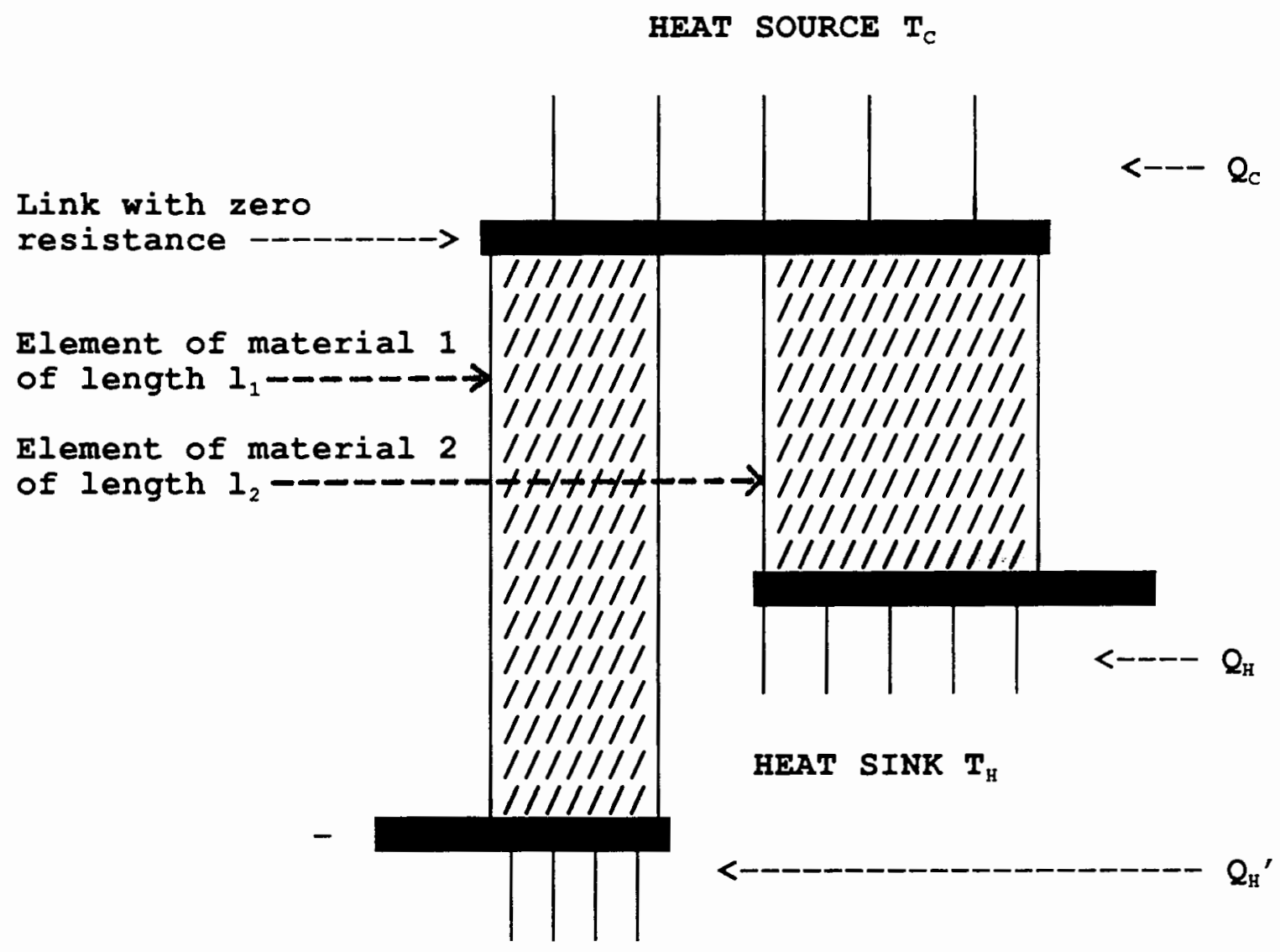

Figure 2. Simple thermoelectric refrigerator.

The rate of heat flow within one of the conductors $\mathrm{i}(\mathrm{i}=1$ or 2$)$ at a distance $\mathrm{x}$ from the heat source is given by

$$
q_{i}=\mp \alpha_{i} I T-\kappa_{i} A_{i} \frac{d T}{d x}
$$


$\mathrm{T}$ is here the absolute temperature over the plane at $\mathrm{x}$ and the Peltier heat flow has been expressed as $\alpha_{\mathrm{i}} \mathrm{IT}$ using Kelvin's first law, Equation (1.4). The rate of heat generation per unit length due to Joule heating is given by

$$
\frac{I^{2}}{\sigma_{i} A_{i}}=-\kappa_{i} A_{i} \frac{d^{2} T}{d x^{2}}
$$

This second order differential equation must now be solved using the boundary conditions $T(x=0)=T_{C}$ and $T\left(x=l_{i}\right)=T_{H}$. The solution yields

$$
q_{i}=\frac{I^{2}\left[x-\left(l_{i} / 2\right)\right]}{\sigma_{i} A_{i}}-\frac{\kappa_{i} A_{i}\left(T_{H}-T_{C}\right)}{l_{i}}
$$

The cooling power $\mathrm{q}_{\mathrm{c}}$ at the heat source is obtained by adding the two contributions $\mathrm{q}_{1}$ and $\mathrm{q}_{2}$ at $\mathrm{x}=0$. It is assumed that the current flows in the correct sense through the thermoelectric element in order to produce cooling rather than heating of the cold side. If it is furthermore assumed that $\alpha_{1}>\alpha_{2}$ then the cooling power is

$$
q_{C}=\left(\alpha_{2}-\alpha_{1}\right) I T_{C}-\frac{I^{2} R}{2}-K\left(T_{H}-T_{C}\right)
$$

where the total electrical resistance of the two branches in series has been defined as

$$
R=\frac{l_{1}}{A_{1} \sigma_{1}}+\frac{l_{2}}{A_{2} \sigma_{2}}
$$

and where the total thermal conductance of the two branches in parallel is given by

$$
K=\frac{A_{1} \kappa_{1}}{l_{1}}+\frac{A_{2} \kappa_{2}}{l_{2}}
$$


Figure 3 shows qualitatively the relation between the cooling power in units of the maximal power and the current through the Peltier element in arbitrary units Io.

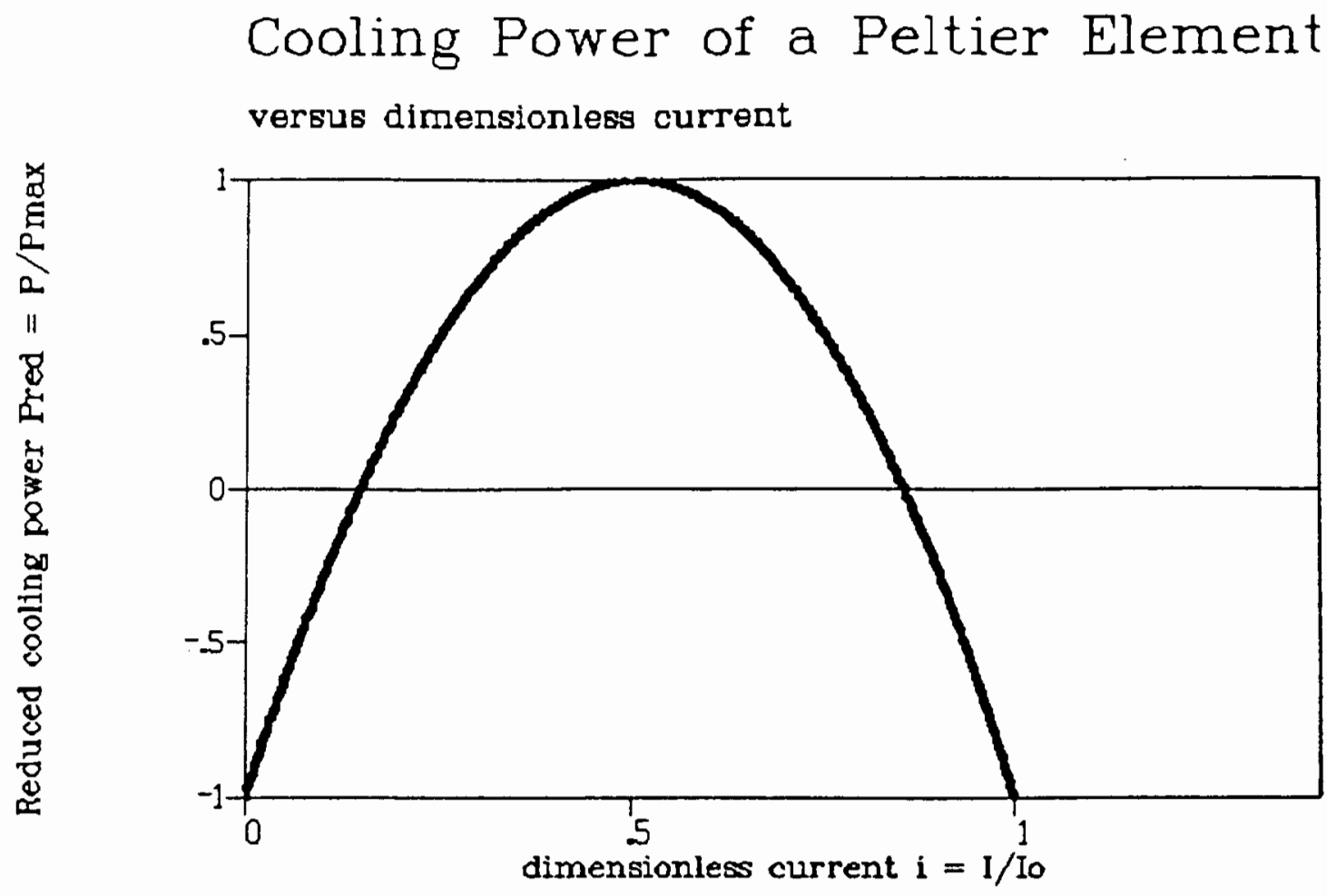

Figure 3. Cooling power of a Peltier element versus dimensionless current.

The total electrical power needed can be shown to be

$$
W=\left(\alpha_{2}-\alpha_{1}\right) I\left(T_{B}-T_{C}\right)+I^{2} R
$$

From equations (1.9) and (1.10) the COP, $\phi$, is found to be

$$
\phi=\frac{\left(\alpha_{2}-\alpha_{1}\right) I T_{C}-I^{2} R / 2-K\left(T_{H}-T_{C}\right)}{\left(\alpha_{2}-\alpha_{1}\right) I\left(T_{H}-T_{C}\right)+I^{2} R}
$$


Figure 4 shows qualitatively the relation between the coefficient of performance (COP) in units of the maximum COP and the current in arbitrary units of Io.

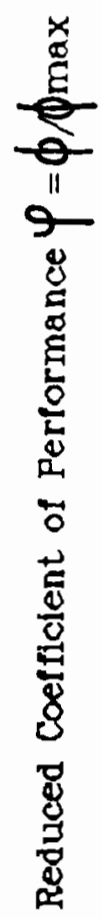

\section{Reduced Coefficient of Performance} versus dimensionless current

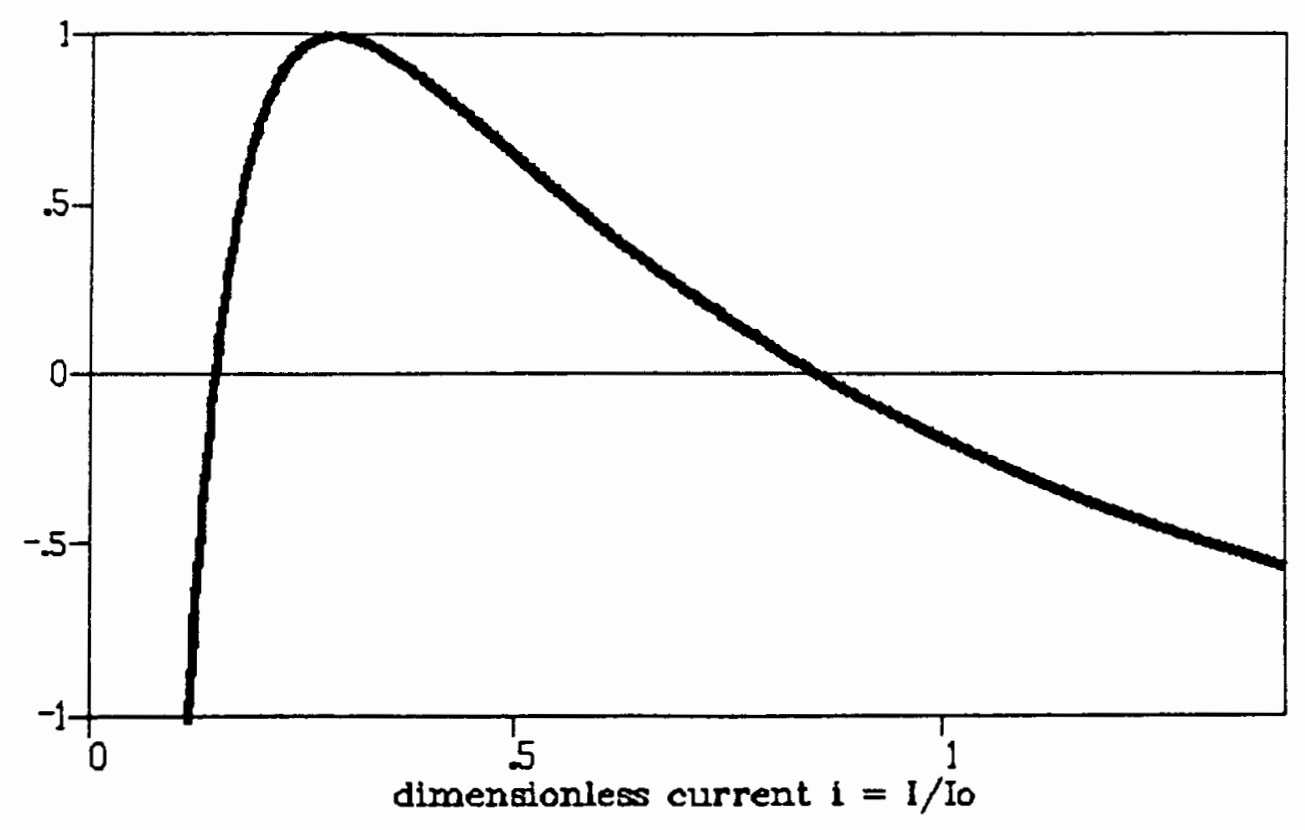

Figure 4. Reduced coefficient of performance of a Peltier element versus dimensionless current.

While the above derivations follow the treatment given by Goldsmid (3), further details are provided by Zemansky and Dittman (4). 


\section{CHAPTER II}

\section{THERMOELECTRICITY}

\section{DESCRIPTION OF THERMOELECTRIC EFFECTS}

The first discovery of a thermoelectric effect took place in 1821 when Thomas Seebeck realized that an electromotive force could be produced by heating a junction between two dissimilar metals. Thirteen years later the French scientist Jean Peltier discovered that the passage of an electric current through the junction of two dissimilar metals caused cooling of one side and in 1838 , Heinrich Lenz was actually able to freeze a droplet of water using the Peltier effect. In 1855, William Thomson derived the relationship between these two effects from thermodynamic principles and he predicted another thermoelectric effect, that had then been unknown.

Referring to Figure 1, the three thermoelectric coefficients will now be defined.

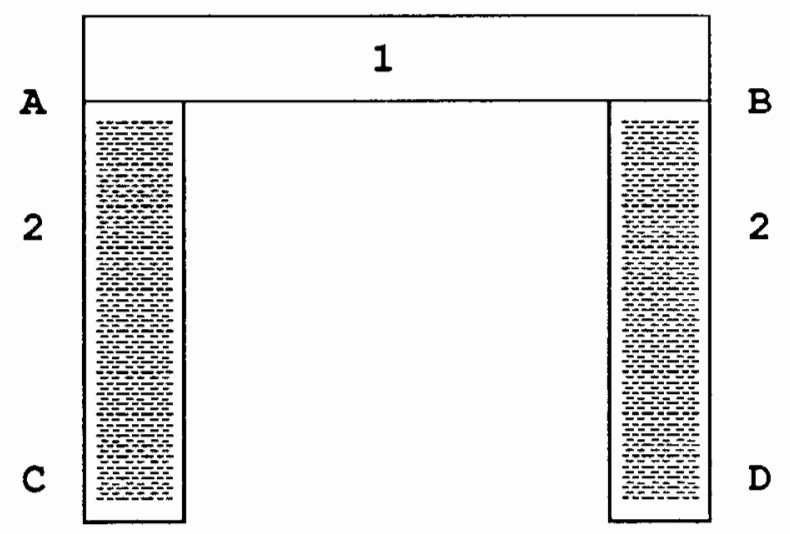

Figure 1. Circuit used in defining the thermoelectric coefficients. 
temperature of $135^{\circ} \mathrm{C}$. Consequently, the whole set-up had to be designed and dimensioned in such a way that the experiment could run during relatively long periods of time (on the order of magnitude of several hours).

Figure 5 shows the set-up of the experiment. It consists of three Peltier elements, two blocks of stainless steel, and four aluminum-blocks on two of which about 30 inches of $\mathrm{NiCr}$-wire are wound thus providing an electric heater. The main part is the Peltier element 2 (PE2), imbedded between two blocks of stainless steel. In each of these blocks there are two thermocouple heads which serve a double purpose: First, they allow one to measure the heat fluxes going to and coming from the thermoelectric element PE2, and secondly, on each side, the knowledge of the temperatures at two points together with the knowledge of the heat flux allows one to extrapolate the temperature directly at the surface of the Peltier element. The experiment can be operated in two different modes. The first mode or standard mode is the case when the Peltier element PE2 actually pumps heat against the temperature gradient and when there consequently is a positive coefficient of performance. The second mode or reverse mode is the case when the selected cooling power is too small to pump heat against the chosen temperature difference, so that heat conduction prevails and consequently the coefficient of performance is negative. In the standard mode of operation, the Peltier element PE1 together with the air cooling provides the heat sink, and the $\mathrm{NiCr}$-wire heating $\mathrm{H} 2$ represents the heat source. In the case when a very large temperature difference and small input power are selected the heat flow is reversed and hence the element PE3 acts as heat sink and the heater $\mathrm{Hl}$ is the heat source. 


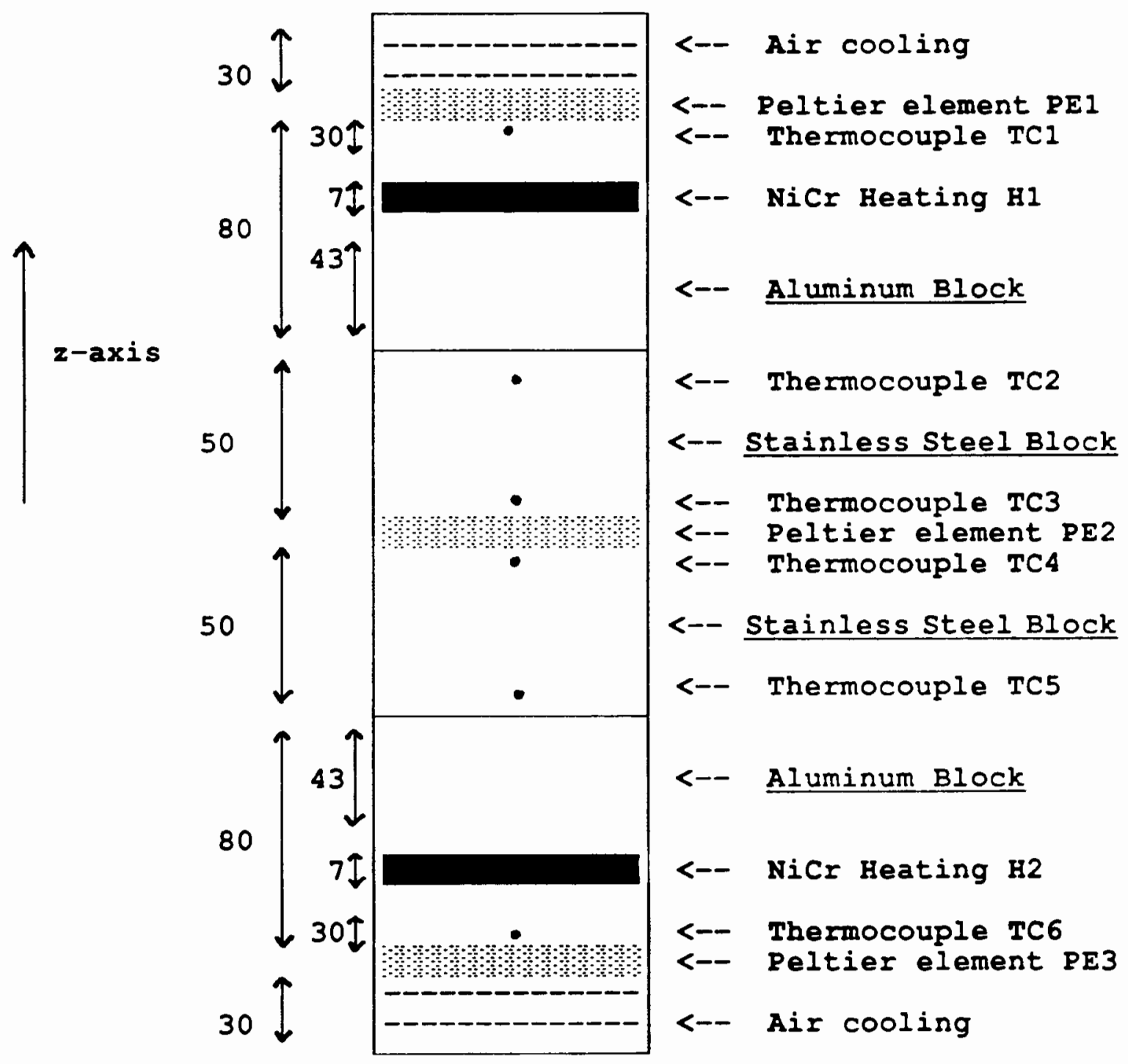

Figure 5. Schematic diagram of the experimental set-up. All length measures are in millimeters. 


\section{PRINCIPLE OF HEAT FLOW MEASUREMENT}

The chosen method of measuring the heat flows going to and coming from the Peltier element PE2 is based on the fundamental law of heat conduction:

$$
\frac{d Q}{d t}=-\kappa A \nabla T
$$

In the equation above $\mathrm{dQ} / \mathrm{dt}$ is the heat flow, $\kappa$ the thermal conductivity, $\mathrm{A}$ denotes the cross-sectional area of the body through which the heat flow is to be determined, and $\mathrm{T}$ is the absolute temperature. Given the knowledge of the temperatures at two points in the center axis of the stainless steel blocks, the determination of the heat flow going to and coming from the Peltier element PE2 rests on the following approximations: First, it is assumed that the temperature gradient in the steel blocks has vanishing radial components and is constant over the whole cross-section, meaning that the surfaces of equal temperature are planes with normals parallel to the center axis. Secondly, it is assumed that the temperature depends linearly on the z-coordinate in order to be able to compute the z-component of the gradient from temperature values at two points of the center axis. Then the equation of heat conduction may be simplified to

$$
\frac{d Q}{d t}=-\kappa A \frac{\Delta T}{\Delta z}
$$

The validity of the first assumption is not trivial, since the heat source, the NiCr-wire heater is wound around a circularly turned circumference at the outer surface of the aluminum block, but could be obtained to some degree of accuracy by the appropriate dimensioning of the blocks and the suitable choice of materials. Since the NiCr-wire 
heater is at a distance of more than twice the diameter of the metal blocks from the first thermocouple tip and since the heat conductivity of aluminum is very high (about 160 $\mathrm{W} / \mathrm{mK}$ ), the temperature gradient in the steel block may be assumed to be homogeneous to a sufficient degree of accuracy. Of course, the above assumptions rely furthermore on negligible heat losses to the lateral surfaces of the metal blocks which is achieved through appropriate insulation.

\section{THERMOCOUPLES}

The accurate measurement of temperatures inside the metal blocks without considerably influencing the heat flows is not a trivial task. Thermistors, for instance, which are otherwise excellent devices for temperature measurements with high precision are definitely too big to be used in the present experiment. Thermocouples seemed to be the most appropriate choice and among them the type $\mathrm{K}$ (Chromel-Alumel) was preferred because of a practically constant Seebeck coefficient over the temperature range of interest. In order to avoid the usual but rather inconvenient ice point reference, a configuration was chosen in which the reference junctions are close together in a large aluminum block of high thermal mass. The basic thermocouple configuration is shown in Figure 6.

Naturally the temperature of the location of the reference junctions varies due to changes in the ambient temperature but a so-called software compensation is performed to account for temperature changes of the reference junctions. The software compensation consists of the temperature measurement at the reference junctions via a thermistor and 
the adjustment of the measured temperatures with the help of a computer subroutine. In this case, particularly small dimensions of the temperature measurement device are not required so that a thermistor is absolutely appropriate for this task. To measure the temperature difference on either the hot or the cold side of the Peltier element, a configuration is used as shown in Figure 7. This has the advantage of a direct, more accurate measurement of the temperature difference as opposed to two single temperature measurements where errors propagate when taking the difference.

\section{TEMPERATURE MEASUREMENTS WITH THERMISTORS}

The temperature measurement with a thermistor is based upon the fact that its resistance $\mathrm{R}$ varies with the temperature $\mathrm{T}$ according to the Steinhart-Hart equation:

$$
\frac{1}{T}=A+B \ln R+C(\ln R)^{3}
$$

where $\mathrm{A}, \mathrm{B}$, and $\mathrm{C}$ are constants which have to be determined for each individual thermistor. For the present experiment the coefficients were determined by a least square fit to ten data points taken at temperatures between $0^{\circ} \mathrm{C}$ and $100^{\circ} \mathrm{C}$ which was performed with a computer program that had to be designed specifically to this purpose. While thermistors could not have been used for the temperature measurements in the metal blocks because of their relatively large size, for the purpose of reference temperature measurement thermistor were preferred since it is a more stable device with fewer sources of error. A detailed description of different temperature measurement devices and methods can be found in reference (5). 


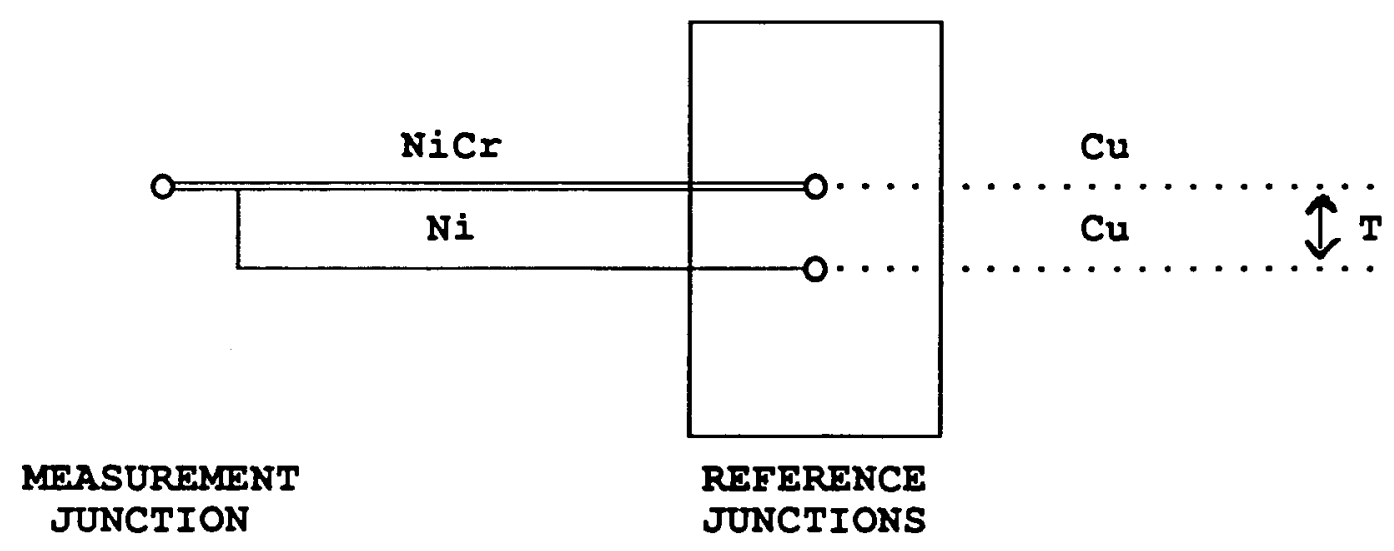

Figure 6. Basic configuration of the type- $\mathrm{K}$ thermocouple.

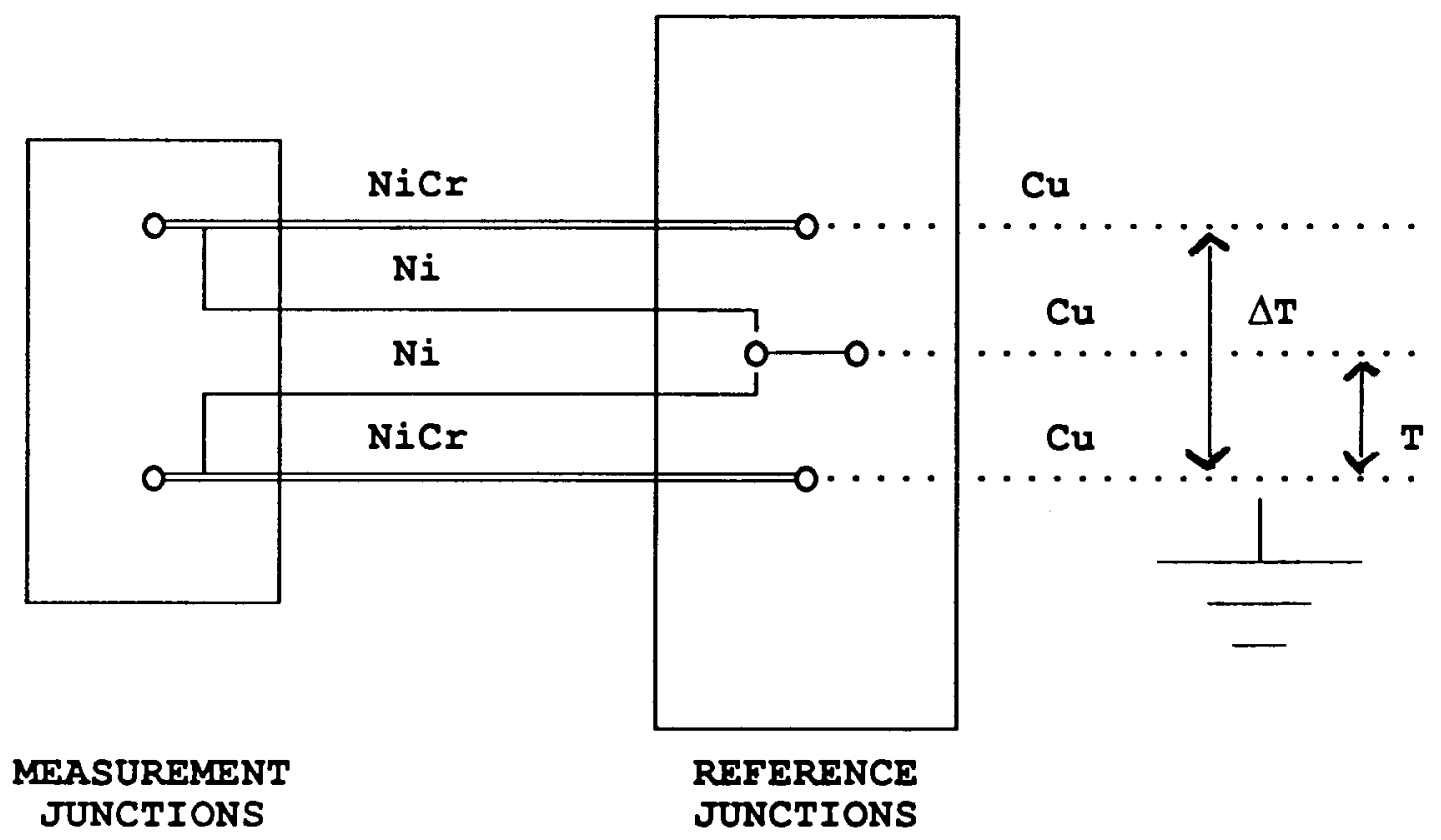

Figure 7. Thermocouple configuration to measure temperature differences. 


\section{ELECTRONIC CIRCUITRY}

Purpose of the control unit

In order to perform the experiment it is necessary to establish a steady state for each selected combination of electrical input power and temperatures on the faces of the Peltier element under investigation. Since there are quite a few parameters to monitor, namely the temperatures at six locations, the input voltage and current of the Peltier element, and the temperature at the reference junction for the thermocouples, a manual operation of the experiment is hardly possible. Therefore some kind of an automatic control unit had to be designed and the most viable way seemed to be the use of a microcomputer with an installed data acquisition system board (DASB). The DASB offers eight differential or sixteen single-ended analog input channels as well as two analog and two digital output channels. Since five loads have to be regulated, namely the three Peltier elements and the two NiCr-wire heaters, the parallel printer port of the computer is used as a third digital output channel. In order to transform the signals of the digital outputs into analog signals necessary to drive the regulation devices, digital to analog converter microchips had to be installed and configured according to the requirements of the loads. To this end, 8-bit converters of the type DAC 0808 are used. Figure 8 shows the structural diagram of the whole control unit.

\section{Description of the components of the control unit}

Input side. Since the voltages produced by thermocouples are of an order of magnitude of tens of microvolts, it is necessary to amplify their output signals in order 


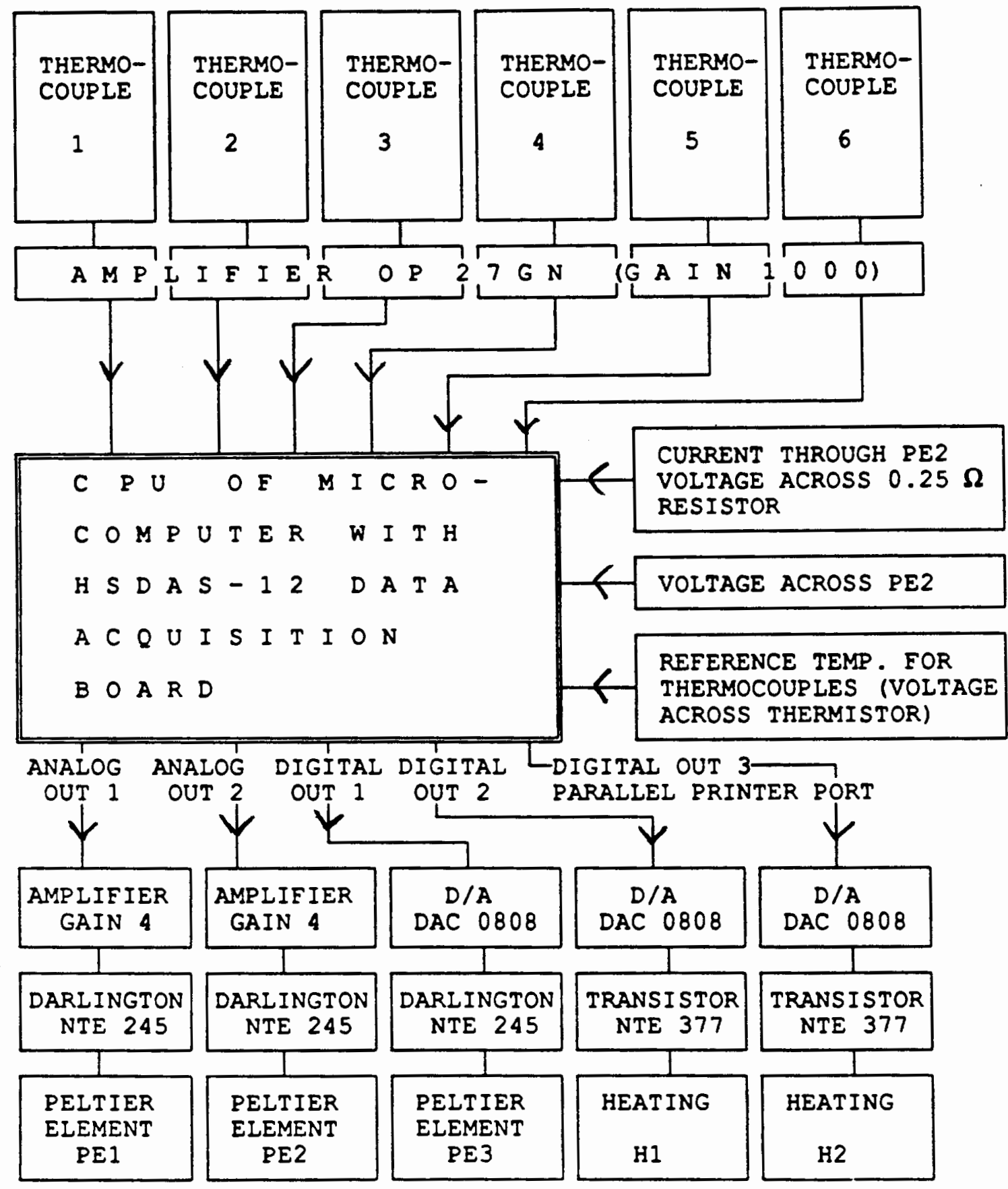

Figure 8. Schematic diagram of the control unit. 
to reach the voltage range that is well resolved by the DASB. An amplification with a voltage gain of 1000 was achieved by employing operational amplifiers of the type OP 27GN in non-inverting application. This microchip has particularly satisfactory low-noise features which is relevant given the considerable voltage gain. To shield the amplification circuits from interference, especially from the line frequency noise, all the amplification circuitry is enclosed in an aluminum case. The inverting inputs of the amplifiers are connected to ground via a $1 \mathrm{~K} \Omega$ resistor and the feedback resistor which connects the amplifier's output with its inverting input is of resistance $1 \mathrm{M} \Omega$. Parallel to the feedback resistor there is a $0.1 \mu \mathrm{F}$ capacitor which serves the purpose of suppressing highfrequency noise. Since the impedance of a capacitor decreases with increasing frequency, the feedback channel is virtually shorted out for high frequencies which leads to a great negative feedback and consequently there is small gain at high frequencies. The temperature at the reference junction is monitored by observing the voltage drop across a thermistor through which a fixed current of $5 \mu \mathrm{A}$ from a constant-current source is flowing. With the resistance of the thermistor being about $1000 \Omega$ at room temperature, the resulting voltage of roughly $5 \mathrm{mV}$ needs to be amplified by a factor of one thousand as well. The other two input signals are already in the appropriate voltage range. The voltage across the Peltier element PE2 can directly be fed into an analog input channel, and the corresponding current is obtained by measuring the voltage drop across a $0.25 \Omega$ resistor. Figure 9 shows the schematic diagram of the amplifier circuit used.

Output side. The Peltier elements used are rated at a maximum voltage of nine volts and at a maximum current of four amps. If measurements at the maximum 


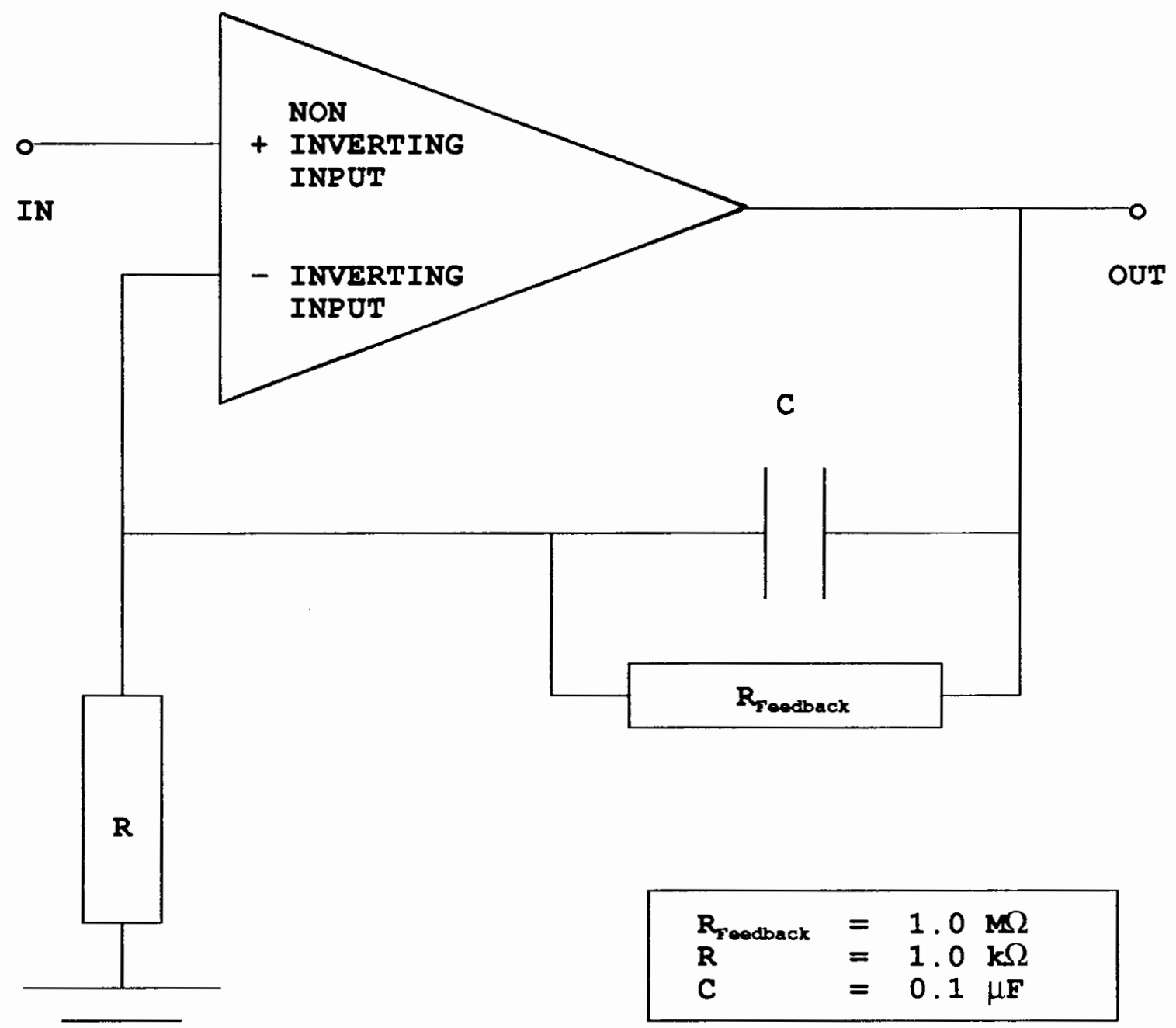

Figure 9. Schematic diagram of the amplifier circuit. 
permissible power range are to be carried out, regulation devices are required that are well able to handle considerable power ranges. Therefore power transistors were chosen to drive all the five loads mentioned above. For the three Peltier elements Darlington pairs of the type NTE 245 were selected because they provide sufficient current gain to be driven by the DASB analog output or the output signal of the digital to analog converters of type DAC 0808 . The two NiCr-wire heaters are driven by power transistors NTE 377.

\section{COMPUTER PROGRAM}

The computer program which has been written in TURBO PASCAL serves the purpose of organizing the monitoring and control of the experiment and of directing the data acquisition. There are three main parts:

(1) Selection of parameters and initialization of the system.

(2) Start of the operation of the Peltier element and beginning of data acquisition.

(3) Continuous monitoring and controlling of the experiment

In the first part the program user selects the values of the temperatures on the faces of the Peltier element and the electric input power and hereby determines the operation mode: Standard or reverse mode. In the standard mode, the electric input power is high enough to actually pump heat against the temperature gradient, whereas in the reverse mode, the cooling power is not sufficiently large relative to the temperature difference in order to overcome the heat conduction "down" the temperature gradient. The mode determines obviously which of the elements providing heat sinks and heat sources, respectively, come into action. 
As a next step, some initial values for the start of the Peltier elements and the NiCr-wire heater are set. After the start of their operation the program organizes a constant measurement of the experimental parameters, performed by the data acquisition board. With the measured temperature data an extrapolation is carried out that determines the temperatures directly on the surfaces of the Peltier element. In order to prevent any of the elements from melting, a protection procedure is included that gives optical and acoustical warning as soon as a certain threshold temperature is reached and exits the program.

The central core of the program is the iteration performed by the procedure Calcvoltage which calculates the power input in the various elements of the experiment based upon the discrepancy between target values and current values. If some parameter $\mathrm{x}$ is to be regulated to a target value $\mathrm{x}_{0}$ and if $\mathrm{x}$ depends on a control parameter $\mathrm{s}$, where $\mathrm{dx} / \mathrm{ds}<0$, then the program contains the following line:

$$
s=s_{0}+\alpha\left(x-x_{0}\right)
$$

where $s_{0}$ is the value of $s$ necessary to keep the system in a steady state when $\mathrm{x}$ is at its target value and where $\alpha$ is a positive scaling parameter. In order to avoid oscillations or even chaotic responses of the system damping has been introduced in the form of time delay commands. The program listing can be found in the appendix.

\section{COMPARISON WITH THE PASCO EFFICIENCY APPARATUS}

About half a year after the work on the present project had begun, the PASCO scientific company presented a lab experiment package titled 'PASCO Efficiency 
Apparatus' which is designed to demonstrate the efficiency of a heat engine or a heat pump using the Peltier effect as an instructive example. Naturally, comparisons between the present experiment and the PASCO package were inevitable and the main differences shall be briefly discussed here. Whereas the PASCO apparatus measures the temperatures of the aluminum blocks with which the Peltier element is in contact via a thermistor, the present experiment was able to give extrapolated values directly on the surfaces of the Peltier element and thus achieves a closer approach to investigating the real efficiency of the Peltier semiconductor elements. Secondly, the present experiment is able to independently establish the temperatures of the hot and cold temperature reservoirs, respectively, and the electrical input power, which enables one to examine the functional relation between efficiency and input current and between cooling power and input current. For the goals of the present project the PASCO efficiency apparatus is not quite flexible enough although it represents a compact student lab experiment that is relatively easy to use and that is well designed to conceptualize the second law of thermodynamics. 


\section{CHAPTER IV}

\section{RESULTS AND CONCLUSIONS}

The goal of the project was to design and to develop an experiment to investigate the performance characteristics of a Peltier refrigeration device operating between two temperature reservoirs. The electrical power input and the temperatures of the reservoirs were to be selected and controlled independently of each other. While this goal could be achieved and a functional set-up has been built, there are still several refinements and improvements necessary in order to obtain results of a sufficient accuracy. The control unit and the computer program proved to be fully operational. However, due to problems with the temperature measurements and with the data acquisition hardware, only a few series of measurements could be performed so far. Among these problems were the intermittent occurrence of absurd temperature measurements, the permanent interference of the control output for the Peltier element PE2 with the thermocouple output voltages, the inexplicably high offset drifts of the amplifier circuits, and the repeated break-down of the central processing unit of the micro computer that was used for the control of the experiment. Because of these difficulties the measurements performed so far did not make use of the full potential of the experiment. The results were obtained by simply setting fixed voltages for the two Peltier elements PE1 and PE2 and the heating device H2 and by taking data after the system had reached a steady state. Through this procedure only a limited temperature range could be examined and a typical measurement 
gave the following result:

Temperature of the hot reservoir:

$37.0^{\circ} \mathrm{C}$

Temperature of the cold reservoir:

$8.3^{\circ} \mathrm{C}$

Cooling power:

$1.1 \mathrm{~W}$

Electrical input power:

$0.33 \mathrm{~W}$

Measured coefficient of performance:

This coefficient of performance (COP) of a refrigeration cycle operating between a hot reservoir at temperature $T_{H}$ and a cold reservoir at temperature $T_{C}$ can be compared to the traditional quasistatic Carnot COP, given by

$$
C O P_{\text {Carnot }}=\left[\frac{T_{H}}{T_{C}}-1\right]^{-1}
$$

and to the COP that Joe Walters (PSU) established as an upper bound on the refrigerator efficiency at maximum cooling power.

$$
C O P_{\text {maximum power }}=\left[\tau^{2} \frac{T_{H}}{T_{C}}-1\right]^{-1}
$$

where

$$
\tau=1+\sqrt{2}
$$

At the given temperatures the Carnot efficiency has a value of 9.8 and a $\mathrm{COP}_{\text {maximum power }}$ of 0.18 is obtained. While it is not surprising that the measured COP lies well below the quasistatic Carnot $\mathrm{COP}$, the amazing fact that it exceeds $\mathrm{COP}_{\text {maximum power }}$ by an order of magnitude is due to the operation of the Peltier element PE2 far below its 
point of maximal power during the measurements performed up to now.

The last paragraph shall be devoted to mentioning possibilities to increase the potential of the experiment and the validity of the measurements that can be taken with its help. A first step to improve upon the experiment is, of course, to check and to correct the temperature measurement part of the experiment. To achieve further enhancement of the experiment it is necessary to verify the assumptions made with respect to the heat flow in the metal blocks carrying the thermocouples. A numerical solution of the heat conduction equation with the specific boundary conditions imposed by the experimental set-up will be useful and such a solution might show that, with the present selection of length dimensions, the assumption of a homogeneous temperature gradient with zero radial components in the metal bodies does not hold to a sufficient degree of accuracy. A third step will be to improve upon the insulation on the sides of the metal blocks and upon the thermal contact on the boundaries between two blocks in order to reduce heat losses and inhomogeneities. To conclude it may be said that the present experiment readily allows to be improved, expanded, and built upon in order to do the further work necessary to a better understanding of thermoelectric energy conversion systems in the context of finite time thermodynamics. 


\section{REFERENCES}

(1) Curzon, F.L. and Ahlborn, B. 1975. Am.J.Phys. 43, 22.

(2) Walters, Joseph D. 1990. "Optimization and Theoretical Performance Measures for a Class of Finite Time Thermodynamical Cycles".

(3) Goldsmid, H. J. 1964. "Thermoelectric Refrigeration", Plenum Press, 1-7.

(4) Zemansky, Mark W. and Dittman Richard H. 1981. "Heat and Thermodynamics", Sixth Edition, McGraw-Hill, 431-442.

(5) OMEGA Engineering, Inc. 1987. "Complete Temperature Measurement Handbook and Encyclopedia". 


\section{APPENDIX}

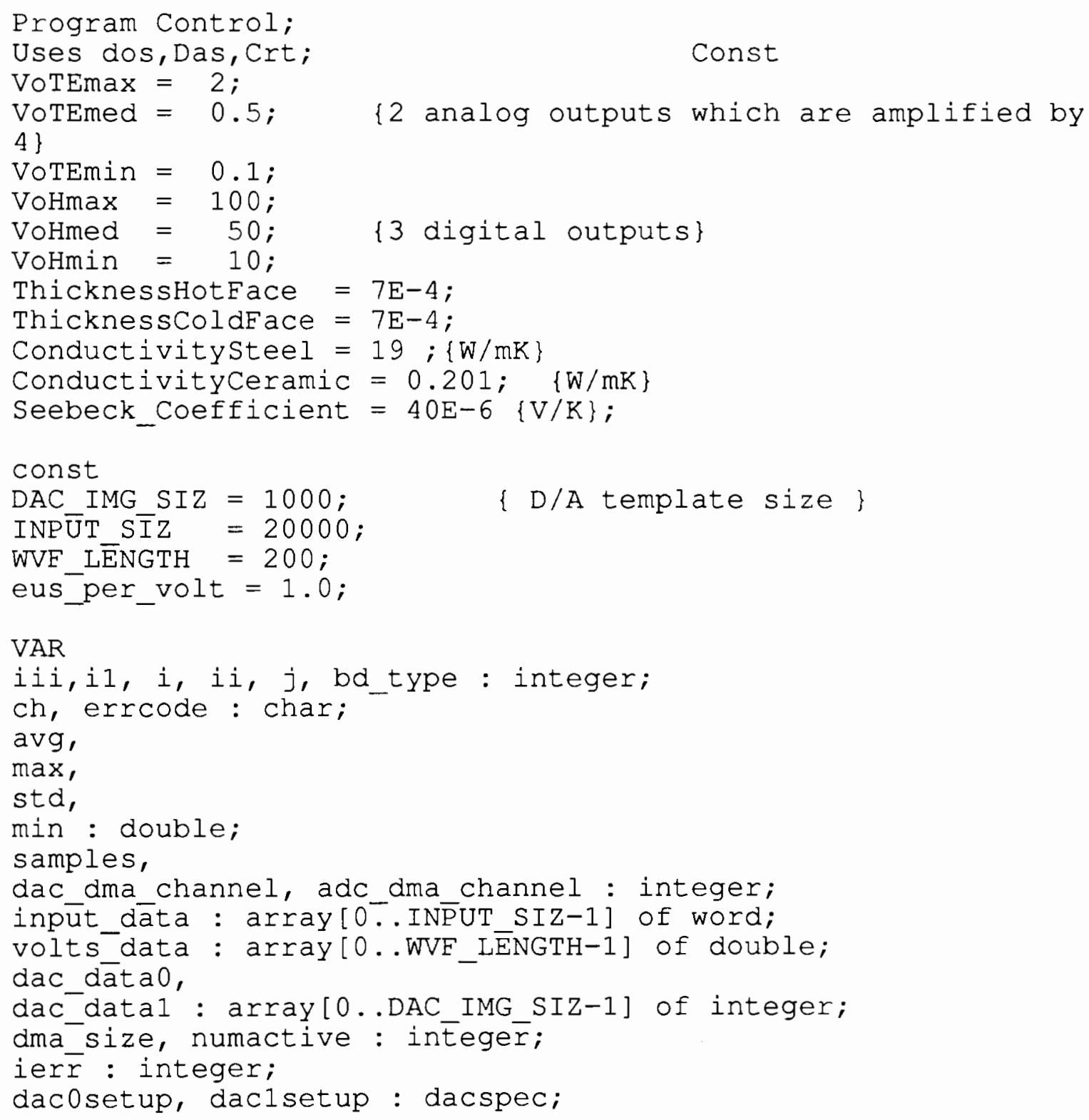


Discrepancytype $=$

(M100,M50,M20,M10,M5, M4,M3,M2,M1,M05,M04,M03,M02, $\mathrm{M} 01, \mathrm{P} 0, \mathrm{P} 01, \mathrm{P} 02, \mathrm{P} 03, \mathrm{P} 04, \mathrm{P} 05, \mathrm{P} 1, \mathrm{P} 2, \mathrm{P} 3, \mathrm{P} 4, \mathrm{P} 5, \mathrm{P} 10, \mathrm{P} 20$,

$\mathrm{P} 50, \mathrm{P} 100)$;

$\{\mathrm{M}$ stands for minus, $\mathrm{P}$ for $\mathrm{Plus,e.g.} \mathrm{M} 100=-100$;

P02 stands for 0.2 - this was necessary because of

restrictions in identifier definitions in Turbo Pascal\}

Type OpAmptype $=$ Record Use :String[50];

Gain : Real;

Offset : Real;

END;

Var

Amplifier : Array[1..8] of OpAmptype;

Scale

Mode

: Scaletype;

Device

: Modetype;

tmp, Pcooling, Icooling,

Vcooling : Real;

Temphot, Tempcold : Array[C..K] of Real;

\{Temphot is the desired temperature\}

fon the hot face of the TE element\}

Tref

: Real;

Vo, VoConst, V,a : Array[H1..TE3] of Real;

Vocurrent, Vlcurrent: real;

Temperature

DataArray

AverageData

Discrepancycode

Discrepancytype;

TenMeasArray

digH2current, $y$ : Longint;
: Array[1..16,1..4] of Real;

: Array[1..8] of Real;

: Array[hotFace..coldFace] of

Procedure SetTemphot;

BEGIN

Write('Enter Temphot in degrees Celsius '); Read(Temphot[C]);

END;

Procedure SetTempcold;

BEGIN

write('Enter Tempcold in degrees celsius ') ; Read (Tempcold [C]);

END;

Procedure SetPcooling;

BEGIN

Write ('Pcooling $=$ '); Read (Pcooling);

END;

Function CtoK(CelsiusTemp:Real):Real;

Var KelvinTemp : Real;

BEGIN

KelvinTemp $:=$ CelsiusTemp + 273.15;

CtoK := KelvinTemp; 
END;

Procedure HeatCoolmode;

BEGIN

Writeln('Standard Mode requires TE1, TE2, and H2');

Writeln(' Mode := Standard') ;

Mode := Standard;

END;

Procedure TEMPREF;

$\operatorname{Var} \mathrm{R}, \mathrm{A}, \mathrm{B}, \mathrm{C}$ : Real;

Eunction Thermistor:Real;

Var $F$ : Real;

Begin

$\mathrm{F}:=\mathrm{A}+\mathrm{B}^{\star} \mathrm{LN}(\mathrm{R})+\mathrm{C}^{\star} \mathrm{LN}(\mathrm{R}) \star \mathrm{LN}(\mathrm{R}) \star \mathrm{LN}(\mathrm{R}) ;$

Thermistor : = $1 / \mathrm{F}$;

end;

BEGIN
$\mathrm{A}:=1.04 \mathrm{E}-3$;
$\mathrm{B}:=3.69 \mathrm{E}-4 ;$
$\mathrm{C}:=-6.9 \mathrm{E}-7$;
R :

(AverageData [2]-Amplifier[2]. Offset)/(Amplifier[2].gain*5E-6);

Tref := Thermistor - 273.15;

END;

Procedure InitVo;

Var VoltageTE, VoltageH : Real;

procedure determination;

BEGIN

If Temphot [C] $>50$ then BEGIN

VoltageTE := VoTEmax;

VoltageH $:=$ VoHmax;

END

else BEGIN

if Temphot [C] $>40$ then BEGIN

VoltageTE := VoTEmed;

VoltageH $:=$ VoHmed;

END

else

BEGIN

VoltageTE := VoTEmin;

VoltageH := VoHmin;

END;

END;

END; fof subprocedure determination\}

BEGIN \{of Procedure InitVo\}

determination;

CASE Mode of Standard : BEGIN

$\mathrm{V}[\mathrm{TE} 1]:=1.2$;

$\mathrm{V}[\mathrm{TE} 2]:=0.8$; 
$\mathrm{V}[\mathrm{H} 2] \quad:=70$;

END;

Plow dThigh : BEGIN

END; There some other determination is

necessary; to be added later

END; \{CASE\}

Vocurrent $:=0$;

Vicurrent: $=0$;

DIGH2 current : $=0$;

END; of Procedure InitVo\}

Procedure Gaugeopamps;

Begin

With Amplifier[1] do begin

Use $:=$ 'Coolingcurrent via $\mathrm{R}=0.25$ ohms; amp not used';

Gain : : 1014;

Offset : $=58$;

end;

With Amplifier[2] do begin

Use $\quad:=$ 'Reference Temperature via thermistor';

Gain : : 1025 ;

Offset $:=46.1$;

end;

With Amplifier[3] do begin

Use $:=$ 'Temperature close to TE1;Protection of TE1';

Gain : : 990;

Offset : $=450$;

end;

With Amplifier[4] do begin

Use $\quad:=$ 'Thot via thermocouple';

Gain : : 958;

Offset $:=250$

end;

With Amplifier[5] do begin

Use $:=$ 'Temperature Gradient on hot side';

Gain $:=1027.3$;

Offset $:=480$;

end;

With Amplifier[6] do begin

Use $:=$ 'Temperature Gradient on cold side' ;

Gain : $=1017.7$;

Offset $:=380$;

end;

With Amplifier[7] do begin

Use $:=$ 'Tcold via thermocouple';

Gain $:=987.6$;

Offset : $=91$;

end;

With Amplifier[8] do begin

Use $:=$ 'Coolingvoltage across TE2 - amp not used ';

Gain : $=992$; 
Offset $:=61$;

end;

for $i:=1$ to 8 do

Amplifier [i] .offset:=Amplifier[i] .offset*1E-3;

END;

procedure digout (bincode:Integer);

Var Printerportchoice : Integer;

$\mathrm{x}$ : Byte;

Begin

Printerportchoice $:=888$;

Port [Printerportchoice] :=bincode;

End;

Procedure SetVolt (Device:Devicetype; voltage:Real);

BEGIN;

If Device $=$ TE2 then BEGIN

DAOUT (voltage, V1current);

vocurrent := voltage;

END;

If Device $=$ TEl then BEGIN

DAOUT (V0current, voltage);

Vlcurrent $:=$ voltage;

END;

If Device $=$ H2 then BEGIN

$\mathrm{y}:=$ trunc (voltage);

Digout $(y)$;

digH2current : $=\mathrm{y}$;

END;

END;

Procedure ConvertData_Temp;

BEGIN

tempref;

Temperature [MP $5, \mathrm{C}]$

Amplifier [3].Offset)/

(Seebeck_coefficient * Amplifier[3].gain) + Tref;

\{Protection of TE1\}

Temperature [MP $2, \mathrm{C}]$

Amplifier [4].Offset)/

(Seebeck_coefficient * Amplifier[4].gain) + Tref;

\{Thot\}

Temperature $[M P 1, C]:=$ Temperature $[M P 2, C]+$

(AverageData[5] - Amplifier[5].Offset)/

(Seebeck_coefficient * Amplifier[5].gain);

\{dThot\}

Temperature [MP 3, C]

Amplifier[7].Offset)/ 
(Seebeck_coefficient * Amplifier[7].gain) + Tref;

$\{\mathrm{Tcold}\}$

Temperature $[\mathrm{MP} 4, \mathrm{C}]:=$ Temperature $[\mathrm{MP} 3, \mathrm{C}]+$

(AverageData[6] - Amplifier[6].Offset)/

(Seebeck_coefficient * Amplifier[6].gain);

end;

Procedure ExtrapolateThot;

Var gradient, Tsurface,gradientCeramic : real;

procedure CalcGradients;

BEGIN

gradient

$:=\quad$ (Temperature $[\mathrm{MP} 2, \mathrm{C}] \quad-$

Temperature $[M P 1, C]) / 0.04 ;\{\mathrm{K} / \mathrm{m}\}$

$\{0.04 \mathrm{~m}$ is the distance between the 2 thermocouples\}

gradientCeramic $:=$ (Conductivitysteel/ConductivityCeramic) * gradient;

END;

BEGIN \{of Procedure ExtrapolateThot\}

CalcGradients;

Tsurface $:=$ Temperature $[M P 2, C]+$ gradient * $5 E-3$

;

Temperature [hotFace, $C]:=$ Tsurface + gradientCeramic *

ThicknessHotFace;

END; \{of Procedure ExtrapolateThot\}

Procedure ExtrapolateTcold;

Var gradient, Tsurface, gradientCeramic : real;

procedure CalcGradients;

BEGIN

gradient

Temperature $[M P 3, \mathrm{C}]) / 0.04 ;\{\mathrm{K} / \mathrm{m}\}$

gradientCeramic $:=$ (ConductivitySteel/ConductivityCeramic) * gradient;

END;

BEGIN \{of Procedure Extrapolatecold\}

CalcGradients;

Tsurface

$:=$ Temperature $[M P 3, C]$ - gradient *

$5 \mathrm{E}-3$;

Temperature [coldFace, $C] \quad:=$ Tsurface - gradientCeramic *

ThicknessColdFace;

END; \{of Procedure Extrapolatecold\}

Procedure Protection;

BEGIN \{of Procedure Protection\}

If Temperature[hotFace,C] $>90$ then BEGIN

Setvolt (TE2,0);

Setvolt $(\mathrm{H} 1,0)$;

Setvolt $(\mathrm{H} 2,0)$;

Sound (220);

Writeln;

Highvideo; 
Write ('PROTECTION came into');

Write ('action ! Temperature at TE2 ');

Writeln('over 90 degrees Celsius');

Writeln;

Delay (1500);

NoSound;

Lowvideo;

Halt;

END;

If Temperature[MP5,C] $>80$ then BEGIN

Setvolt $(\mathrm{H} 1,0)$;

Sound (220);

Writeln;

Highvideo;

Write ('PROTECTION came into');

Write ('action! Temperature at TE1 ');

Writeln('too high');

Writeln;

Delay (1500);

NoSound;

Lowvideo;

Halt;

END;

If Temperature $[M P 6, C]>80$ then BEGIN

Setvolt $(\mathrm{H} 2,0)$;

Sound (220);

Writeln;

Highvideo;

Write ('PROTECTION came into');

Write ('action! Temperature at $\mathrm{TE} 3$ ');

Writeln('too high');

Writeln;

Delay (1500);

NoSound;

Lowvideo;

Halt;

END;

END; \{of Procedure Protection\}

Function Get_Discrepancy (location:MPtype):Real;

BEGIN

If location $=$ hotFace THEN

Get Discrepancy := Temperature[hotFace, C] - Temphot [C];

If Iocation = coldFace THEN

Get_Discrepancy := Temperature[location,C] - Tempcold[C];

END $\bar{i}$

Procedure CodeAssignment (location:MPtype);

Var C : Discrepancytype;

$G$ : Real;

Begin 
G := Get Discrepancy (location);

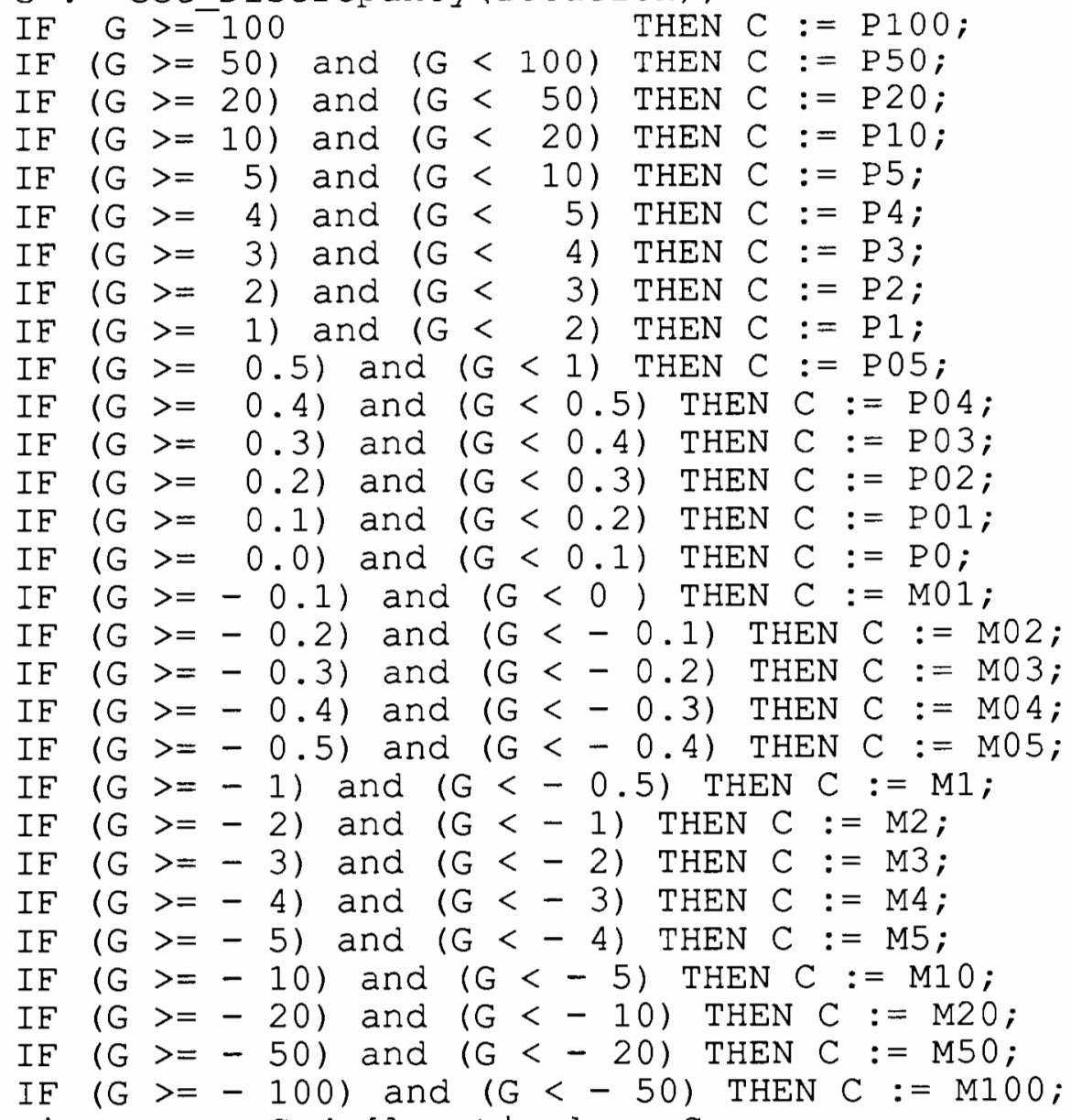

DiscrepancyCode[location] := C;

END;

Function Resistance:Real;

BEGIN

tmp:= Vcooling/Icooling;

If tmp<0.1 then tmp:=3;

resistance: $=$ tmp;

END;

Function Power:Real;

BEGIN

tmp: = Vcooling * Icooling;

if tmp $<0.1$ then tmp: $=0$;

power: =tmp;

END;

PROCEDURE GET TEMPERATURES;

var

location:MPtype;

BEGIN 
$\{$ Now measure inputs with the adc \}

ierr := adinit ('last.cfg');

if ierr $<0$

then begin

case ierr of

-14: writeln('Could not find DAS configuration file "LAST. CFG"');

else

writeln('ADC initialization failed due to error \# ', ierr); end;

exit

end;

$\{$ count up the number of active channels \}

numactive : $=0$;

for $i:=0$ to 15 do

numactive $:=$ numactive + ((das_cfg_table.storage_enable

shr i) AND 1);

dma_size:=trunc(numactive * das_cfg_table.samples_per_chan); if dma_size > INPUT_SIZ

then dma_size := INPUTT_SIZ;

if numactive $=0$

then begin

writeln('No channels were activated - data collection aborted');

exit

end

else samples $:=$ dma_size div numactive;

delay (1000);

lowvideo;

ierr := adpoll (@input_data, dma_size);

data $\}$

if ierr $<0$

Then BEGIN \{ service any errors \}

case ierr of

-5: Writeln('Time out occured before data collection was complete');

-7: Writeln('Detected FIEO overflow - aborted data collection');

-22: Writeln('Detected ESC key being pressed - user aborted data collection');

end;

Exit

End;

I Now convert the data to voltages, then display the data summary\}

for $j:=0$ to 15 do

if (das cfg table.storage enable shr j) AND $1 \ll 0$ )

THEN \{ if data was taken \}

BEGIN

$\{$ convert data to voltages \} 
ierr := gtdat(@input_data, j+1, @volts_data, eus_per_volt, samples);

avg $:=0.0 ;$

std $:=0.0$

$\max :=$ volts data $[0] ;$

min:= volts_data[0];

for $i i:=0$ to samples -1 do begin

avg: $=$ avg+volts data[ii];

if $\max <$ volts_data[ii]

then $\max :=$ volt $\bar{s}$ data [ii]

else if min > volts data[ii]

then min:= volts_data[ii]

end;

avg:= avg/samples;

AverageData $[j+1]:=a v g$;

if samples $>2$

then begin

for $i \mathrm{i}:=0$ to samples-1 do

std: = std+ (avg - volts data[ii])*(avg - volts data[ii]);

std: $=\operatorname{sqrt}($ std / samples $)$

end;

writeln(' channel ', $(j+1): 2,{ }^{\prime}:{ }^{\prime}, \operatorname{avg}: 10: 6,{ }^{\prime}{ }^{\prime}, \max : 10: 6,{ }^{\prime}$ ', $\min : 10: 6,{ }^{\prime}$ ', std: $\left.10: 6\right)$;

END;

delay (1); delay (1);

writeln;

writeln(' Vocurrent (voltage across TE2) ',V0current:10:6);

writeln(' Vlcurrent (voltage across TE1) ',Vlcurrent:10:6);

writeln(' digH2current (digital signal to H2) ', digH2current);

ConvertData_Temp;

Icooling :=-AverageData $[1] * 4$;

Vcooling := AverageData[8] - AverageData[1];

If (Icooling<0.0) and (Vcooling<0.0) then Vcooling:=0;

ExtrapolateThot;

ExtrapolateTcold;

writeln('hotface ', temperature[hotface, C]:4:2,' coldface

', temperature [coldface, C]:4:2);

$i:=0$;

writeln; writeln('tref ', tref:4:2);

for location:=MP 1 to MP5 do

begin

$i:=i+1$;

writeln (i,' ', temperature [location, $c]: 4: 2, '$

volt

', averagedata $[i+2]: 6: 4)$;

end;

writeln('power ', power);

Protection;

END;

Procedure CalcVoltage; 
BEGIN

If (DiscrepancyCode[hotface] < M10) or (Discrepancycode [hotFace] > P10)

THEN BEGIN

$\mathrm{V}[\mathrm{TE} 1] \quad:=\mathrm{V}[\mathrm{TE} 1]+0.01 *$ (Temperature [hotFace, $\mathrm{C}]$ Temphot [C]);

END

ELSE BEGIN

$\mathrm{V}[\mathrm{TE} 1] \quad:=\mathrm{V}[\mathrm{TE} 1]+0.001 *$ (Temperature[hotEace, $\mathrm{C}]-$ Temphot [C]);

END;

If (DiscrepancyCode[coldFace] < M10) or (DiscrepancyCode [coldFace] > P10)

THEN BEGIN

$\mathrm{V}[\mathrm{H} 2]:=\mathrm{V}[\mathrm{H} 2]-10 \star$ (Temperature $[\mathrm{coldF} a c e, \mathrm{C}]-$ Tempcold $[\mathrm{C}])$; END

ELSE BEGIN

$\mathrm{V}[\mathrm{H} 2]:=\mathrm{V}[\mathrm{H} 2]-1 \star$ (Temperature $[\operatorname{coldFace}, \mathrm{C}]-\mathrm{Tempcold}[\mathrm{C}]) ;$ END;

If Pcooling $>$ Power

THEN V[TE2] $:=\mathrm{V}[\mathrm{TE} 2]+0.01$ \&QRT $($ (PCOOling - Power $)$ *

Resistance)

ELSE V[TE2] $:=\mathrm{V}[\mathrm{TE2}]-0.01$ * SQRT((Power - Pcooling) *

Resistance);

if $\mathrm{V}[\mathrm{H} 2]>200$ then $\mathrm{V}[\mathrm{H} 2]:=200$;

if $\mathrm{V}[\mathrm{Te} 2]>2$ then $\mathrm{V}[\mathrm{Te} 2]:=2$;

if $\mathrm{V}[\mathrm{Te} 1]>2$ then $\mathrm{V}[\mathrm{Te} 1]:=2$;

For Device $:=\mathrm{H} 1$ to TE3 do BEGIN

If $\mathrm{V}$ [device] < 0 THEN $\mathrm{V}$ [device] $:=0$;

END;

END; $\{$ CalcVoltage

Procedure Minor;

BEGIN

writeln ('M i $\left.\mathrm{n} \circ \mathrm{r}^{\prime}\right)$;

For il $:=1$ to 10 do BEGIN

delay (2000);

Get Temperatures;

end;

CalcVoltage;

SetVolt (TE1, V[TE1]);

Setvolt (H2, V[H2]);

END;

Procedure Major;

BEGIN

Writeln ('M a jo r');

Get Temperatures;

Calc̄Voltage;

SetVolt (TE1, V[TE1]);

Setvolt (TE2, V[TE2]); 
Setvolt (H2, V[H2]);

END;

BEGIN \{of main program\}

gaugeopamps;

bd type :=\$FF;

err̄code := char $(0)$;

\{ Tell the driver where the das board is mapped, also resets board \}

ierr := bdinit $(0$, bd_type, 5, 6);

if (ierr < 0) THEN

BEGIN

WRITELN(' DAS board could not be initialized. ');

case ierr of

-1: Writeln('No DAS board found at address', das cfg table.board address);

-5: Writeln ('Could not calibrate board');

-6: Writeln('Illegal DMA channel specified');

-14: Writeln('No DAS configuration file - "LAST.CEG" was found.' ) ;

end;

Exit;

End;

stmo (100);

\{ Set dma timeout \}

SetPcooling;

Set Temphot;

Set Tempcold;

HeatCoolMode;

Initvo;

Setvolt (TE1, V[TE1]);

Setvolt (TE2, V[TE2]);

writeln ('test'); Setvolt (H2,V[H2]);

for iii:=1 to 2 do get temperatures;

REPEAT Minor; delay (2000);

Major;

UNTIL KEYPRESSED;

END. 